\title{
Exploring the mechanism of astaxanthin against lipopolysaccharide-induced acute lung injury by network pharmacology and experimental validation
}

lianxiang luo ( $\square$ luolianxiang321@gdmu.edu.cn )

Guangdong Medical College Zhanjiang Campus: Guangdong Medical University https://orcid.org/0000-0002-3391-9713

Xiaoling Li

Guangdong Medical College Zhanjiang Campus: Guangdong Medical University

Riming Huang

South China Agricultural University

Hui Luo

Guangdong Medical College Zhanjiang Campus: Guangdong Medical University

Research

Keywords: Acute lung injury, Astaxanthin, TLR4, MyD88, NF-kB

Posted Date: March 23rd, 2021

DOI: https://doi.org/10.21203/rs.3.rs-334157/v1

License: (c) (i) This work is licensed under a Creative Commons Attribution 4.0 International License. Read Full License 


\section{Abstract}

\section{Background}

Acute lung injury (ALI) is a leading cause of morbidity and mortality in respiratory disease. Astaxanthin, a natural antioxidant xanthophyll carotenoid, has been shown to possess anti-inflammatory activity. However, poor evidence has been reported that whether it has protective effects against ALI.

\section{Methods}

A network pharmacology analysis was carried out combining the construction of the GeneCards database and the Pharmmapper database, The potential active compounds and targets were predicted by compound-target prediction, protein-protein interaction analysis, GO and KEGG pathway analysis. Then, the anti-inflammation effect of astaxanthin was investigated in LPS-induced RAW264.7 cells in vitro and LPS-induced ALI mice in vivo.

\section{Results}

The results screened by GO and KEGG enrichment analysis suggested that astaxanthin had extensive associations with 25 known therapeutic targets of ALI. These target genes were further found to be associated with pathways involved in inflammatory pathways in ALI, such as the Toll-like receptor signal pathway, TNF signal pathway, Hif signal pathway, and NF-Kappa B signal pathway. Pre-treatment with astaxanthin inhibited the TLR4/MyD88 signaling pathway and attenuated LPS-increased inflammatory factors in vitro. Furthermore, the administration of astaxanthin significantly protected lung injury in vivo. Subsequently, we validated astaxanthin binds to the TLR4 pocket using molecular docking.

\section{Conclusion}

Taken together, astaxanthin exerts impressively protective effects on LPS-induced ALI in vitro and in vivo via suppressing the TLR4/MyD88 signaling pathway.

\section{Introduction}

Acute lung injury (ALI) is the main cause of morbidity and mortality of respiratory diseases, which is characterized by lung oedema, interstitial neutrophilic infiltration, intravascular, and endothelial barriers resulting in respiratory failure [1, 2]. ALI-related mortality remains high [3]. The pathogenesis of ALI is complex, and studies have shown that pulmonary inflammation plays an important role in the progression of the disease, Alveolar macrophage promotes the development and maintenance of pulmonary inflammation by secreting stimulating levels of pro-inflammatory cytokines, such as tumor necrosis factor-a (TNF-a), interleukins (e.g.IL-6, IL-1 $\beta$ )[4]. Unfortunately, there are no proven pharmacological therapies for ALI. Therefore, the identification of effective therapeutic drugs is urgently needed for the improvement of ALI treatment. 
Astaxanthin (3, 3'-dihydroxy- $\beta$, $\beta^{\prime}$-carotene-4, 4'-dione) is a xanthophyll carotenoid, which is mainly distributed in seafood such as shrimp, salmon, crabs and so on $[5,6]$. Astaxanthin has a variety of potential pharmacological activities, including immunomodulation, antioxidants, anti-obesity, antiinflammatory, anti-cancer as well as neuroprotective effects [7-9]. Studies have shown that astaxanthin plays an anti-inflammatory role by decreasing the expression of pro-inflammatory cytokines in alveolar lymphocytes, neutrophils, macrophages [10]. Astaxanthin plays an anti-inflammatory and antioxidant roles in macrophages in NRF2-dependent and independent manners [11]. Astaxanthin attenuated ethanolinduced liver injury by blocking STAT3 activity [12]. Furthermore, astaxanthin inhibited inflammation response and reverse LPS-induced M1/M2 polarization of microglial cells by targeting RP-1 [13]. However, the anti-inflammatory effects and the molecular mechanisms of astaxanthin in the acute lung injury have not been well studied.

Network pharmacology is a new subject of designing drugs based on the theory of systems biology and network analysis of biological systems[14]. Network pharmacology adopts the strategy of "network target, multi-component" to replace the current research method of "one target, one drug" strategy[15]. Because it saves time and costs, it has been considered to be an important tool for drug discovery[16]. The technique has been successfully applied to understand the mechanism of action of several medicinal plants used for treatment of various diseases $[17,18]$. Molecular docking predicts the binding conformation and affinity of drug molecules through the interaction between receptors and drug molecules, which involves the spatial and energy matching between molecules, as an important technology in the field of computer-aided drug research, these two methods provide powerful tools for the study of modern pharmacological mechanism of traditional Chinese medicine.

In this study, in order to illustrate the possible protection mechanism of astaxanthin against ALI, a network pharmacology method coupled with molecule docking was conducted, and provide a reference for further in vitro and in vivo experiments verification, which providing a new insight into potential therapeutic strategies for ALI.

\section{Materials And Methods}

\section{Prediction of target genes}

The 2D chemical structure of astaxanthin was obtained from PubChem, and the astaxanthin-related predicted targets were predicted by the Pharmmapper database. Each of the predicted targets was inputted into the UniProt database (https://www.uniprot.org/) for further screening. The GeneCards database was used to obtain known therapeutic targets for treatment of ALI. The potential target of astaxanthin associated with Ali was obtained by removing the repeated target and deleting the target with a relevance score $<0.001$.

\section{PPI network construction and analysis}


The target genes were uploaded to the STRING database (http://string-db.org) to provide the proteinprotein interaction information, including the physical and functional associations. Protein-protein interactions with confidence (score at $>0.9$ ) were retained in the network. The PPI network was visualized by Cytoscape software.

\section{GO and KEGG pathway enrichment analysis}

The GO and KEGG pathway enrichment analysis were carried out using the web (https://biit.cs.ut.ee/gprofifiler), and visualized by the OmicShare cloud platform (http://www.omicshare.com/). The target-pathway, target-pathway, and compound-target networks were constructed using Cytoscape software.

\section{Molecular docking}

The X-ray crystal structure of the TLR4/MD-2 complex was retrieved from the Protein Data Bank (PDB: 5IJB), and the 3D structure of astaxanthin was obtained from PubChem (compound CID: 5281224). The water molecules and hetero atoms were removed from the proteins using Chimera. The protein receptor and ligand files were performed using AutoDock4 Tools, the hydrogen atoms were added, gasteiger charges were calculated, nonpolar hydrogen atoms were merged, and rotatable torsion bonds were set. The interactions between astaxanthin and the TLR4/MD-2 complex was visualized, 2D and 3D diagrams were created by using Pymol.

\section{Cell culture}

RAW264.7 cells were cultured in high-glucose Dulbecco's modified Eagle's medium (DMEM) supplemented with $10 \% \mathrm{FBS}, 100 \mathrm{U} / \mathrm{ml}$ penicillin and $100 \mu \mathrm{g} / \mathrm{ml}$ streptomycin. The cells were maintained at $37^{\circ} \mathrm{C}$ with $5 \% \mathrm{CO} 2$.

\section{Western blot}

Cells were lysed with RIPA buffer supplemented with PMSF on ice for 10 min. Cell lysates were cleared by centrifugation for $10 \mathrm{~min}$ at $12,000 \mathrm{~g}$. The protein concentration of lysates was detected by BCA assays. Each sample with $20 \mu \mathrm{g}$ of total protein was separated by SDS-PAGE gel and transferred onto NC membranes. Membranes were blocked in 5\% (wt/vol) dried milk in TBS-T for $1 \mathrm{~h}$ at room temperature. Primary antibodies were added to membranes and incubated overnight at $4^{\circ} \mathrm{C}$ with gentle rocking. After washing, blots were incubated with the HRP-conjugated secondary antibody for $1 \mathrm{~h}$ at room temperature. ECL plus system was applied for detection of the protein signal. Antibodies to the following proteins were used in the blots: COX-2 (CST, cat\#12282, 1:1000 dilution), iNOS (CST, cat\#D6B6S, 1:1000 dilution), TLR4(Santa Cruz, cat\# sc-293072, 1:1000 dilution), MYD88(Santa Cruz, cat\# sc-74532, 1:1000 dilution), p-NF-kB p65(CST, cat\#3033,1:1000 dilution), GAPDH (CST, cat\#5174,1:1000 dilution), anti-rabbit IgG (CST, cat\#7074, 1:3000 dilution) and anti-mouse IgG (CST, cat\#5127, 1:3000 dilution).

\section{Immunofluorescence}


Cells were seeded in coverslips and treated with astaxanthin for $1 \mathrm{~h}$ and then stimulated with LPS for $24 \mathrm{~h}$. Cells were fixed for 10 min with $4 \%$ paraformaldehyde, permeabilized for 10 min with $0.3 \%$ Triton X-100 and blocked for $30 \mathrm{~min}$ with $5 \%$ goat serum at room temperature. Cells were incubated with the primary antibodies overnight at $4^{\circ} \mathrm{C}$. Samples were washed and incubated with an anti-mouse IgG fluorescencelabeled secondary antibody for $1 \mathrm{~h}$ at room temperature. After three times washed, samples were stained with DAPI at 1:1000 (Cell Signaling Technology, 4083) for $10 \mathrm{~min}$ at room temperature. Finally, coverslips mounted on slides with fluorescent mounting medium. Images were acquired by a Cytation ${ }^{\text {TM }} 5$ cell imaging multi-mode reader (BioTek).

\section{ALI mouse model}

The animal experiments involved in this experiment were conducted through the Animal Ethics Committee of Guangdong Medical University (GDY1902062), all animal experiments were performed in compliance with institutional animal care guidelines and according to committee-approved protocols. 6week-Babl/c female mice were randomized to the following three groups of seven mice each: vehicle group, LPS group, Astaxanthin plus LPS group. Astaxanthin + LPS group mice were pretreated with astaxanthin $(20 \mathrm{mg} / \mathrm{kg})$ by i.v injection for daily for 7 consecutive days. Astaxanthin was dissolved in $2 \%$ DMSO(vol/vol), 40\%PEG-400(vol/vol), $2 \%$ Tween $80(\mathrm{vol} / \mathrm{vol})$, and $56 \% \mathrm{PBS}(\mathrm{vol} / \mathrm{vol})$. On the last day, the mice were intraperitoneally injected with $5 \mathrm{mg} / \mathrm{kg}$ LPS or normal saline $2 \mathrm{~h}$ after the injection of astaxanthin. After $6 \mathrm{~h}$ of LPS stimulation, mice were euthanized to collect the BALF, and lung tissue samples. BALF was collected three times through a tracheal cannula with autoclaved normal saline, instilled up to a total volume of $1.8 \mathrm{ml}$.

\section{Lung wet-to-dry weight (W/D) ratio}

After mice were euthanized, the lung tissues were yield and weighed, and then dried at $60^{\circ} \mathrm{C}$ for $72 \mathrm{hours,}$ the lung tissues were weighed again. The ratio of lung wet-to-dry weight was calculated.

\section{Determination total protein concentration in BALF}

Bronchoalveolar lavage fluid (BALF) cells supernatant was centrifuged at $3000 \mathrm{rpm}$ for $10 \mathrm{~min}$ at $4 \mathrm{C}$. The total protein of BALF was quantified using a BCA assay kit according to the manufacturer's instructions.

\section{Histopathology and Immunohistochemistry}

Lung tissues were fixed in $10 \%$ formalin, embedded in paraffin. The sections were dewaxed using xylene and dehydrated in a graded series of alcohol solutions. Histological analysis was performed using $\mathrm{H} \& \mathrm{E}$ staining according to a standard protocol. For histology analysis, the sections were stained with hematoxylin and eosin (H\&E). For IHC analysis, paraffin-embedded tissue sections were treated with citrate buffer solution, and treated with microwave for $15 \mathrm{~min}$, and then the temperature was reduced to room temperature. Tissues were incubated with $3 \% \mathrm{H}_{2} \mathrm{O}_{2}$ for 30 min to block endogenous peroxidase 
activity. Subsequently, the sections were incubated with $5 \%$ normal goat serum for 30 min, and incubated with CD68 antibody (CST, cat\#76437,1:1000 dilution) at $4{ }^{\circ} \mathrm{C}$ overnight. The sections were labeled with HRP secondary antibodies for $30 \mathrm{mins}$ at room temperature. The samples were then stained with 3, 3'diaminobenzidine (DAB) for 5-10 min and counterstained with Mayer's hematoxylin. The sections dehydrated, cleared in xylene and mounted in mounting medium. Images were acquired by Cytation ${ }^{\mathrm{TM}} 5$ cell imaging multi-mode reader (BioTek).

\section{Statistical analysis}

The results are presented as the mean $\pm S D$. The statistical significance of differences was assessed using the ANOVA and Student's $t$ test. $P<0.05$ were considered statistically significant.

\section{Results}

\section{GO and KEGG enrichment analysis of target genes}

Astaxanthin-targeted protein-coding genes are involved in biological processes including cell response to oxidative stress, regulation of cell apoptosis, and response to inflammation (figure 1B). The molecular functions of these proteins include protein binding, cytokine receptor binding, receptor ligand activity, receptor regulatory activity, and cofactor binding (figure 1C). The KEGG pathway analysis further indicated that astaxanthin-targeted proteins were involved in the Toll like reception signaling pathway, TNF signaling pathway, HIF signaling pathway and NF-Kappa B signaling pathway (figure 1A). GO and KEGG analysis results demonstrated that the genes encoding astaxanthin- targeted proteins may have the functions of Toll like receptor signal pathway, TNF signal pathway, Hif signal pathway and NF-Kappa B signal pathway (figure 1D). These pathways involve in cytokine receptor and ligands activation.

\section{PPI network constitution and identification of candidate targets for astaxanthin against ALI}

PPI network analysis of astaxanthin anti-ALI target showed that astaxanthin anti-ALI target genes were involved in many biological pathways, such as biological processes, cellular components and molecular functions (figure 2A). Inflammation plays an important role in this process, and the inflammatory response mainly involves the following gene: IL1B, RPS6KA5, HMGB1, IL6, TNF, SPP1, NFE2L2, CXCL8, PTGS2 (figure 2B). Moreover, we further analysis showed that astaxanthin targeted proteins are involved in IL-17 signaling pathway, Chemokine signaling pathway, MAPKsignaling pathway, PI3K-Akt signaling pathway, FoxO signaling pathway, Toll-like receptor signaling pathway, NF-kappa B signaling pathway, TNF signaling pathway, Th17 cell differentiation, Cytokine-cytokine receptor interaction, NOD-like receptor signaling pathway, Jak-STAT signaling pathway, HIF-1 signaling pathway (figure 2C). Therefore, the results showed that astaxanthin can inhibit the production of inflammatory factors through different signaling pathways and cytokine-cytokine receptors interaction.

\section{T-P network analysis of ALI-related pathways}


Previous data showed that targets of astaxanthin are involved in the regulation of inflammation [12]. The results of GO and KEGG enrichment analyses were displayed in figure 3, the target genes were enriched in multiple biological process and pathway, it can be seen that astaxanthin regulates the inflammatory response in acute lung injury mainly through the following four signaling pathways including HIF-1a signaling pathway, TNF-a signaling pathway, JAK-STAT signaling pathway, and Toll-like receptor signaling pathway.

\section{Astaxanthin inhibits LPS-stimulated expression of pro-inflammatory cytokines and NF-KB in RAW264.7 cells}

We determined whether astaxanthin could modulate LPS-induced iNOS and COX-2 expression in RAW264.7 cells. As shown in figure 4B, astaxanthin distinctly suppressed expression of COX-2 and iNOS in RAW264.7 cells induced by LPS with immunoblotting. The nuclear translocation of NF-kB was analyzed by an immunofluorescence assay. As shown in figure 4B囚phosphorylated NF-kB p65 (p-p65) indited that NF-KB was translocated from cytosol to nucleus induced by LPS. Whereas, treatment with astaxanthin effectively blocked the nuclear translocation of NF-KB (figure 4A). These results indicate that astaxanthin decreased LPS-induced inflammatory responses by attenuating the release of proinflammatory cytokines via the TLR4/MyD88 signaling pathway.

\section{Network visualization analysis of anti-inflammatory effect of astaxanthin via TLR4 / myd88 pathway}

In order to build a more systematic inflammatory network, a diagram consisting of inflammatory biomarkers was constructed by cytoscape software. There are different degrees of direct or indirect interaction between node edges in the analysis, inflammatory factor such as TNF-a, IL-6, NF-kB, IL-1b was identified as import targets linking astaxanthin to the TLR4/MyD88 signaling pathway (figure 5A). To further confirm the effect of astaxanthin on the TLR4/MyD88 signaling pathway at the molecular level. As we expected, western blot results showed that astaxanthin effectively suppressed TLR4/MyD88 signaling pathway (figure 5B).

\section{Binding modes of astaxanthin to TLR4/MD-2 complex}

As we known, TLR4 associates with myeloid differentiation factor 2 (MD2) by forming the TLR4/MD2 complex, which recognized by LPS [19]. Thus a molecular docking simulation was performed to predict potential interaction between astaxanthin and the TLR4/MD-2 complex. The result shows that astaxanthin may form conventional hydrogen bonds with side chains of PHE-24 and MET-358, and form carbon-hydrogen bond with LYS-360 of TLR4. Because the sites of PHE-24, MET-358 and LYS-360 are very close to critical sites of TLR4/MD-2 complex (figure 6A and 6B), the results suggest that astaxanthin may inhibit the stable formation of TLR4-MD2 complex, and affect downstream signal pathway of TLR4. 
Pathologic changes of lung tissues were evaluated by H\&E staining. As illustrated in figure 7A, LPS induced several obvious inflammatory histological changes in lung tissues, such as pulmonary congestion, and inflammatory cells infiltration, which were significantly attenuated by astaxanthin treatment. Accordingly, treatment of astaxanthin significantly decreased LPS-induced lung W/D weight ratio (figure 7B). Furthermore, pretreatment with astaxanthin remarkably decreased the number of infiltrated cells (figure 7D) and total protein concentration in BALF (figure 7C), indicating decreased pulmonary edema. Additionally, pretreatment with astaxanthin significantly inhibited LPS-induced lung macrophage cell infiltration into the lung tissue, as evidenced by CD68-immunostaining in figure 7E. Taken together, these data indicate that astaxanthin alleviates LPS-induced ALI in mice.

\section{Discussion}

ALI is known as a lung complication with a high rate of morbidity and mortality [3]. Current pharmacological interventions and therapies have failed to treatment of ALI [20]. Therefore, it is urgent to understand the mechanisms of ALI and develop new therapeutic strategies. The present study demonstrated that astaxanthin inhibit pro-inflammatory cytokine expression in LPS-induced ALI in vitro and in vivo. The proposed mechanism illustration was presented in Fig. 8.

It is well known that network target recognition can be used to be a target prediction of active compounds[21]. Pharmacology analysis based on a large number of existing databases can help people better understand the mechanism of the target and pathway of compounds acting on the corresponding diseases [22]. In our current study, we found that the infection effect plays an important role in this process, and the infectious response mainly involves the following gene: IL1B, RPS6KA5, HMGB1, IL6, TNF, SPP1, NFE2L2, CXCL8, PTGS2. KEGG analysis further showed that four pathways, including HIF-1a signaling pathway, TNF-a signaling pathway, Toll-like receptor signaling pathway and JAK-STAT signaling pathway, were closely associated with astaxanthin against ALI, our results further demonstrated that astaxanthin alleviate LPS-induced ALI by down-regulating the expression of proinflammatory cytokine in vitro and in vivo.

It is well known that TLR4 is expressed on monocyte/macrophage surface and induces innate immune response in mammals through recognition of LPS [23]. LPS induced ALI and activated the TLR4 as well as NF-KB signal pathway to regulate the inflammatory cells infiltration and the pro-inflammatory cytokines production [24-26]. Studies have shown that LPS promotes the excessive production of inflammatory mediators by activating the TLR4/Myd88 signaling pathway, leading to lung damage and ALI progression $[27,28]$. Recent studies have shown that several natural products modulate the inflammatory response by inhibiting the TLR4/NF-KB signaling pathway [29-33]. Our results showed that astaxanthin inhibit the TLR4/MyD88/NF-KB signaling pathway. We further used molecular docking to detect whether astaxanthin can be directly targeted to TLR4, thus inhibiting the TLR4 signaling pathway. The result showed that astaxanthin bound to the hydrophobic pocket of TLR4/MD2 complex, which partially overlapped with the LPS binding sites on TLR4 surface. These data suggested that antiinflammatory activity of astaxanthin through its inhibitory effect on the specific TLR4 signaling pathway. 


\section{Conclusions}

In conclusion, we demonstrated that astaxanthin effectively protected ALI against inflammation damage through regulation of TLR4/MyD88/NF-KB signaling pathway. Our study provided evidence that astaxanthin could be a potential therapeutic candidate for the treatment of ALI in the future.

\section{Abbreviations}

ALI, Acute lung injury; BALF, Bronchoalveolar lavage fluid; COX-2, Cyclooxygenase-2; INOS, H\&E, Hematoxylin and eosin; Inducible nitric oxide synthase; IL-6, Interleukin-6; LPS, Lipopolysaccharide; MD2, Myeloid differentiation factor 2; MyD88, Myeloid differentiation factor 88; NF-kB, Nuclear factor-kB; TNFa, Tumor necrosis factor-a; TLRs, Toll-like receptors.

\section{Declarations}

\section{Acknowledgment}

This project was supported by the PhD Start-up Fund of Guangdong Medical University (B2019016); Administration of Traditional Chinese Medicine of Guangdong Province (20201180); Administration of Traditional Chinese Medicine of Guangdong Province (20211223); Science and Technology Special Project of Zhanjiang (2019A01009); Basic and Applied Basic Research Program of Guangdong Province (2019A1515110201); Key Program of Marine Economy Development(Six Marine Industries) Special Foundation of Department of Natural Resources of Guangdong Province(GDNRC[2020]038); Fund of Southern Marine Science and Engineering Guangdong Laboratory (Zhanjiang) (ZJW-2019-007).

\section{Data Availability}

The data used to support the findings of this study are included within the article.

\section{Declaration of competing interest}

The authors declare that they have no competing interests.

\section{Consent for publication}

Not applicable.

\section{Ethics approval and consent to participate}

All animal experimental procedures were approved by the Animal Care and Use Committee of Guangdong Medical University in line with the guidelines of National and Institutional Guidelines for Animal Care

\section{Author contributions}


LX Luo conceived and designed the experiments; XL Li, LX Luo performed the experiments and analyzed the data; LX Luo and XL Li wrote the manuscript; RM Huang and $\mathrm{H}$ Luo reviewed the paper and provided comments, and all authors reviewed the manuscript.

\section{References}

[1] Ding C, Chen H, Liang B, Jiao M, Liang G, Zhang A. Biomimetic synthesis of the natural product salviadione and its hybrids: discovery of tissue-specific anti-inflammatory agents for acute lung injury. Chem Sci. 2019;10:4667-72.

[2] Spinelli E, Mauri T, Beitler JR, Pesenti A, Brodie D. Respiratory drive in the acute respiratory distress syndrome: pathophysiology, monitoring, and therapeutic interventions. Intensive Care Med. 2020.

[3] Butt Y, Kurdowska A, Allen TC. Acute Lung Injury: A Clinical and Molecular Review. Arch Pathol Lab Med. 2016;140:345-50.

[4] Levy BD, Serhan CN. Resolution of acute inflammation in the lung. Annu Rev Physiol. 2014;76:467-92.

[5] Fakhri S, Abbaszadeh F, Dargahi L, Jorjani M. Astaxanthin: A mechanistic review on its biological activities and health benefits. Pharmacol Res. 2018;136:1-20.

[6] Higuera-Ciapara I, Felix-Valenzuela L, Goycoolea FM. Astaxanthin: a review of its chemistry and applications. Crit Rev Food Sci Nutr. 2006;46:185-96.

[7] Faraone I, Sinisgalli C, Ostuni A, Armentano MF, Carmosino M, Milella L, et al. Astaxanthin anticancer effects are mediated through multiple molecular mechanisms: A systematic review. Pharmacol Res. 2020;155:104689.

[8] Davinelli S, Nielsen ME, Scapagnini G. Astaxanthin in Skin Health, Repair, and Disease: A Comprehensive Review. Nutrients. 2018;10.

[9] Kishimoto Y, Yoshida H, Kondo K. Potential Anti-Atherosclerotic Properties of Astaxanthin. Mar Drugs. $2016 ; 14$.

[10] Cai X CY, Xie X, Yao D, Ding C, Chen M. Astaxanthin prevents against lipopolysaccharide-induced acute lung injury and sepsis via inhibiting activation of MAPK_NF-KB.pdf. Am J Transl Res. 2019;11:1884-94.

[11] Farruggia C, Kim MB, Bae M, Lee Y, Pham TX, Yang Y, et al. Astaxanthin exerts anti-inflammatory and antioxidant effects in macrophages in NRF2-dependent and independent manners. J Nutr Biochem. 2018;62:202-9.

[12] Han JH, Ju JH, Lee YS, Park JH, Yeo IJ, Park MH, et al. Astaxanthin alleviated ethanol-induced liver injury by inhibition of oxidative stress and inflammatory responses via blocking of STAT3 activity. Sci 
Rep. 2018;8:14090.

[13] Wen X XL, Zhong Z, Wang L, Li Z, Pan X, Liu Z. Astaxanthin acts via LRP-1 to inhibit inflammation and reverse lipopolysaccharide-induced M1_M2 polarization of microglial cells.pdf. Oncotarget. 2017;8:69370-85.

[14] AL H. Network pharmacology.pdf. Nat Biotechnol. 2007;25:1110-1.

[15] Boezio B, Audouze K, Ducrot P, Taboureau O. Network-based Approaches in Pharmacology. Mol Inform. 2017;36.

[16] Barabasi AL, Oltvai ZN. Network biology: understanding the cell's functional organization. Nat Rev Genet. 2004;5:101-13.

[17] Zhang W, Huai Y, Miao Z, Qian A, Wang Y. Systems Pharmacology for Investigation of the Mechanisms of Action of Traditional Chinese Medicine in Drug Discovery. Front Pharmacol. 2019;10:743.

[18] Huang C, Zheng C, Li Y, Wang Y, Lu A, Yang L. Systems pharmacology in drug discovery and therapeutic insight for herbal medicines. Brief Bioinform. 2014;15:710-33.

[19] Hajjar AM, Ernst RK, Fortuno ES, 3rd, Brasfield AS, Yam CS, Newlon LA, et al. Humanized TLR4/MD-2 mice reveal LPS recognition differentially impacts susceptibility to Yersinia pestis and Salmonella enterica. PLoS Pathog. 2012;8:e1002963.

[20] Wheeler AP, Bernard GR. Acute lung injury and the acute respiratory distress syndrome: a clinical review. The Lancet. 2007;369:1553-64.

[21] Yildirim MA, Goh KI, Cusick ME, Barabasi AL, Vidal M. Drug-target network. Nat Biotechnol. 2007;25:1119-26.

[22] Hopkins AL. Network pharmacology: the next paradigm in drug discovery. Nat Chem Biol. 2008;4:68290.

[23] Park BS, Lee JO. Recognition of lipopolysaccharide pattern by TLR4 complexes. Exp Mol Med. 2013;45:e66.

[24] Anwar MA, Shah M, Kim J, Choi S. Recent clinical trends in Toll-like receptor targeting therapeutics. Med Res Rev. 2019;39:1053-90.

[25] Hennessy EJ, Parker AE, O'Neill LA. Targeting Toll-like receptors: emerging therapeutics? Nat Rev Drug Discov. 2010;9:293-307.

[26] Keogh B, Parker AE. Toll-like receptors as targets for immune disorders. Trends Pharmacol Sci. $2011 ; 32: 435-42$. 
[27] Zhao G ZT, Ma X, Jiang K, Wu H, Qiu C, Guo M, Deng G. Oridonin attenuates the release of proinflammatory cytokines in lipopolysaccharide-induced RAW264.7 cells and acute lung injury.pdf. Oncotarget. 2017;8:68153-64.

[28] Qi M, Zheng L, Qi Y, Han X, Xu Y, Xu L, et al. Dioscin attenuates renal ischemia/reperfusion injury by inhibiting the TLR4/MyD88 signaling pathway via up-regulation of HSP70. Pharmacol Res. 2015;100:341-52.

[29] Liu Z, Chen L, Yu P, Zhang Y, Fang B, Wu C, et al. Discovery of 3-(Indol-5-yl)-indazole Derivatives as Novel Myeloid Differentiation Protein 2/Toll-like Receptor 4 Antagonists for Treatment of Acute Lung Injury. J Med Chem. 2019;62:5453-69.

[30] Wang N, Geng C, Sun H, Wang X, Li F, Liu X. Hesperetin ameliorates lipopolysaccharide-induced acute lung injury in mice through regulating the TLR4-MyD88-NF-kappaB signaling pathway. Arch Pharm Res. 2019;42:1063-70.

[31] Wu Y, Wang Y, Gong S, Tang J, Zhang J, Li F, et al. Ruscogenin alleviates LPS-induced pulmonary endothelial cell apoptosis by suppressing TLR4 signaling. Biomed Pharmacother. 2020;125:109868.

[32] Liu JX, Li X, Yan FG, Pan QJ, Yang C, Wu MY, et al. Protective effect of forsythoside B against lipopolysaccharide-induced acute lung injury by attenuating the TLR4/NF-kappaB pathway. Int Immunopharmacol. 2019;66:336-46.

[33] Peri F, Calabrese V. Toll-like receptor 4 (TLR4) modulation by synthetic and natural compounds: an update. J Med Chem. 2014;57:3612-22.

\section{Figures}


A

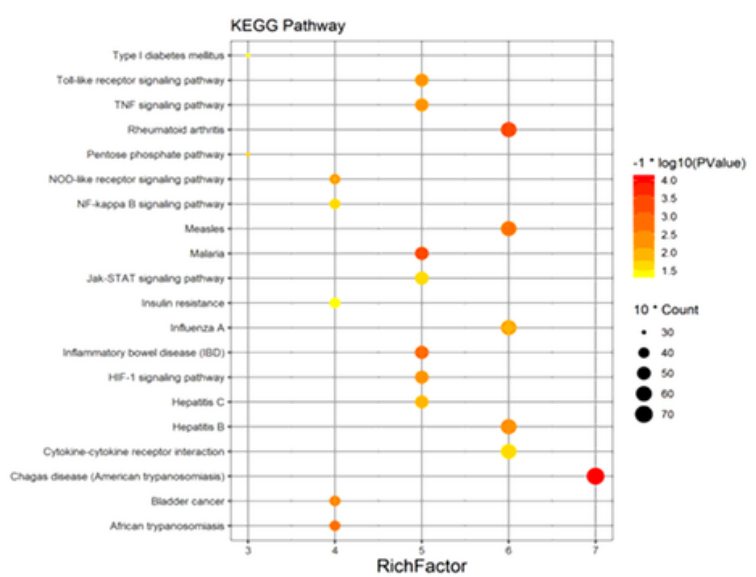

$\mathrm{C}$

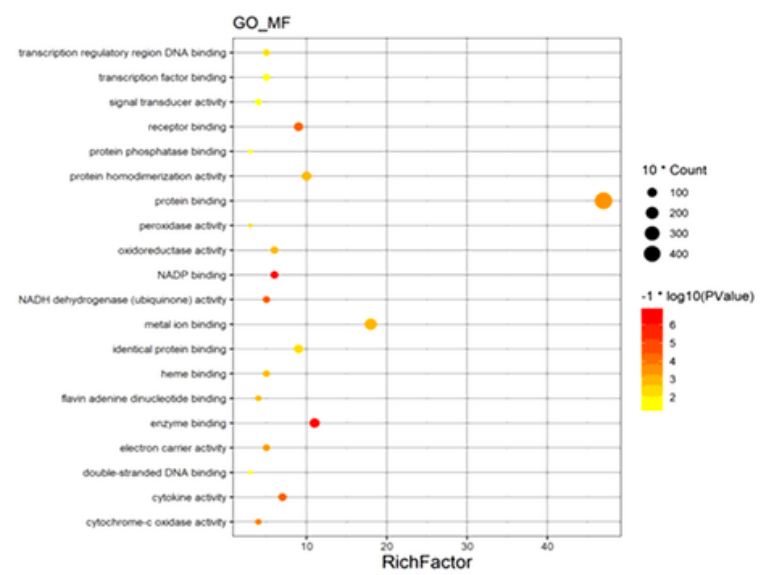

$\mathrm{B}$

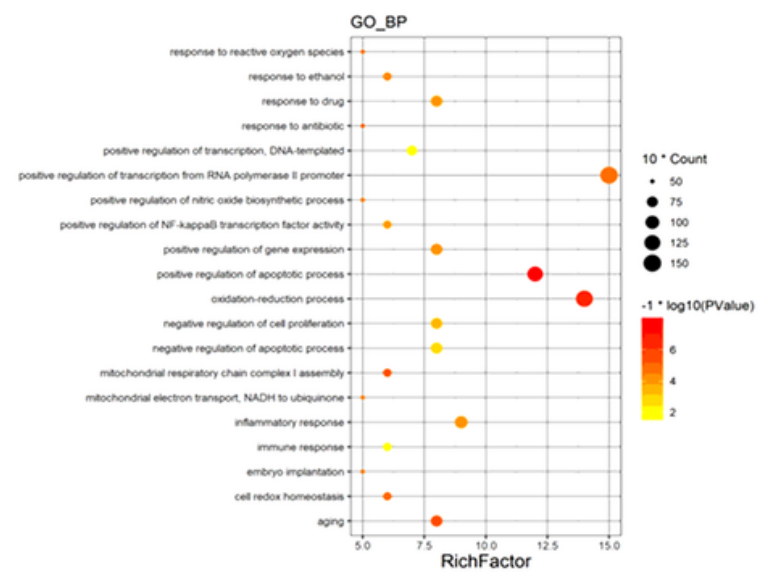

$\mathrm{D}$

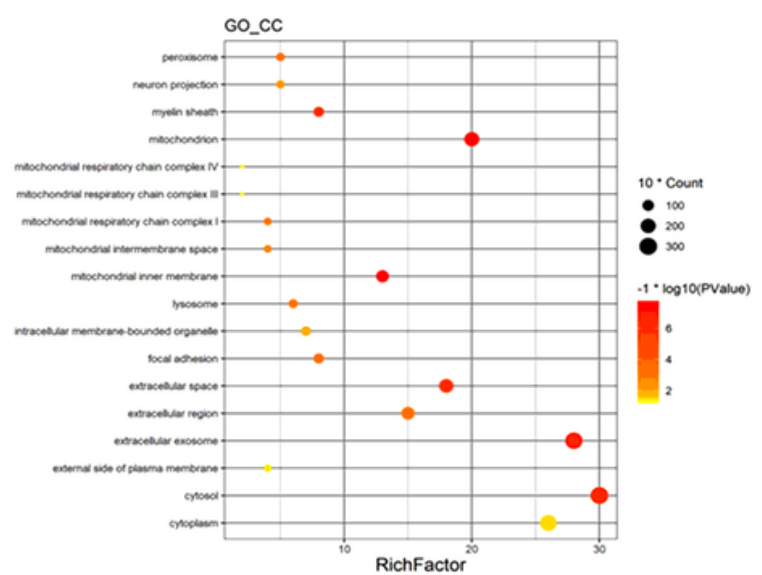

\section{Figure 1}

Kyoto Encyclopedia of Genes and Genomes (KEGG) pathway and gene ontology enrichment analyses of 25 target proteins ( $p$-value $\leq 0.05$ ). (A) The top 20 KEGG pathways. (B) The top 20 biological processes. (C) The top 20 molecular functions. (D) The top 18 cellular components. The color scales indicate the different thresholds for the p-values, and the sizes of the dots represent the number of genes corresponding to each term. 
A

AIFM1 APP CIOA CAT COX5A CTSS CXCL8 CYPIAL ODR1 F7 FOXO3 G6PO GFAP GAA

GNA13 GPX3 GRIN1 GRN GSR GSTM2 HMGB1 HMOX1 HOXA9 IDH1 IFNG LL1B IL6 JAK1

LEPR MMP1 NOUFAI NOUFV2 NFE2L2 NME2 NOS2 NOO1 PAH PGD POR PPP1CB PROX1 PTGS2

RPSGKAS SOO1 SP1 SPP1 STAT3 TGM TKT TNF TNNT2 TPS3 UBB VOCRC2 APP G6PD

GFAP GNA13 GSTM2 HMGB1 ILE NOOI PRDX1 PTGS2 RPS6KAS SO01 STAT3 TNF TP53 UOCRC2

$\mathrm{C}$

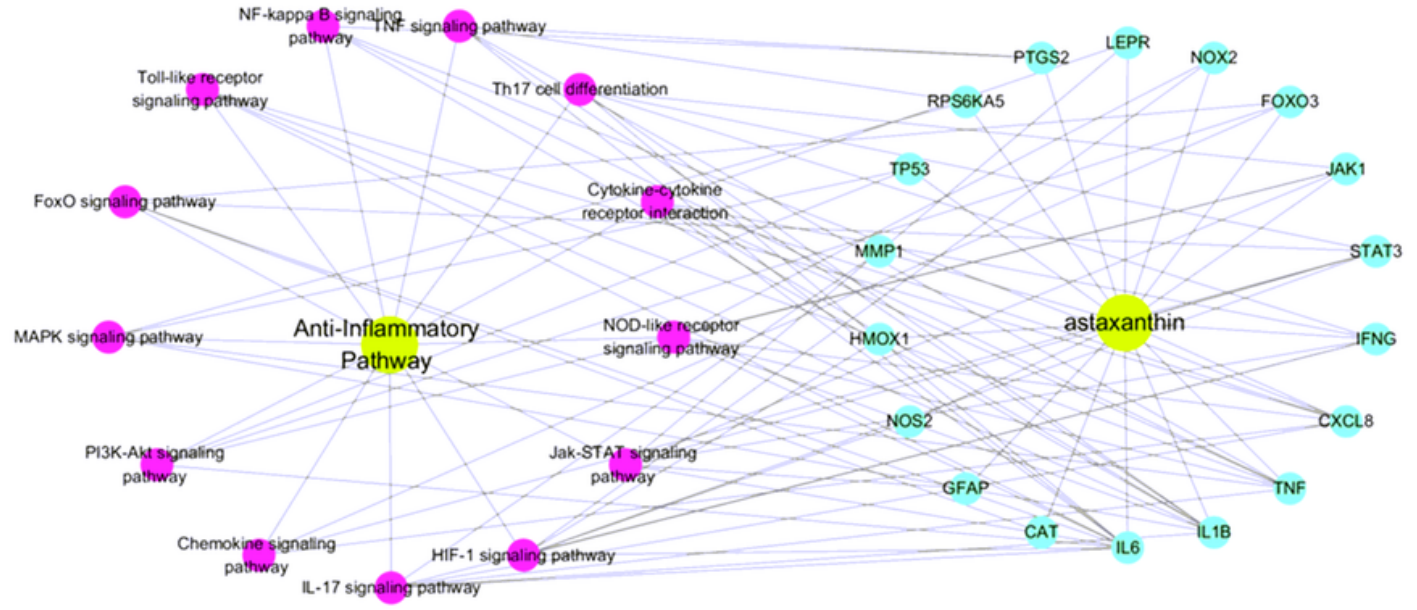

Figure 2

Protein-protein interaction (PPI) network construction and analysis. (A) Core target-pathway network. (B) Identification of candidate targets for astaxanthin against ALI. (C) Drug-compound-target-disease network map 


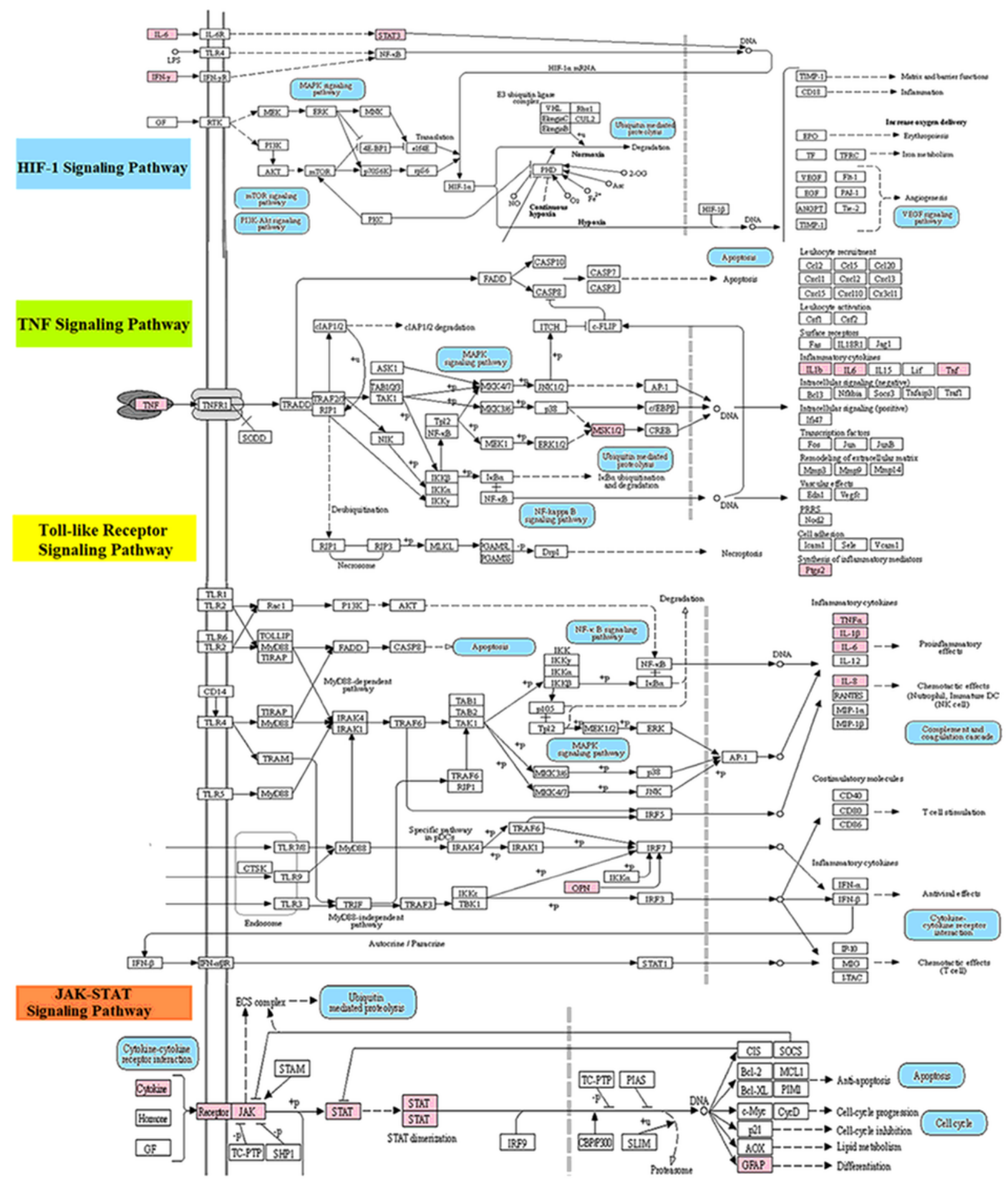

\section{Figure 3}

Distribution of targets of astaxanthin in inflammatory-related pathways. Arrows shows activation effect; T-shaped arrow shows inhibition effect, and dotted arrow represents indirect activation effect or inhibition effect. 
A

LPS(100ng/ml)

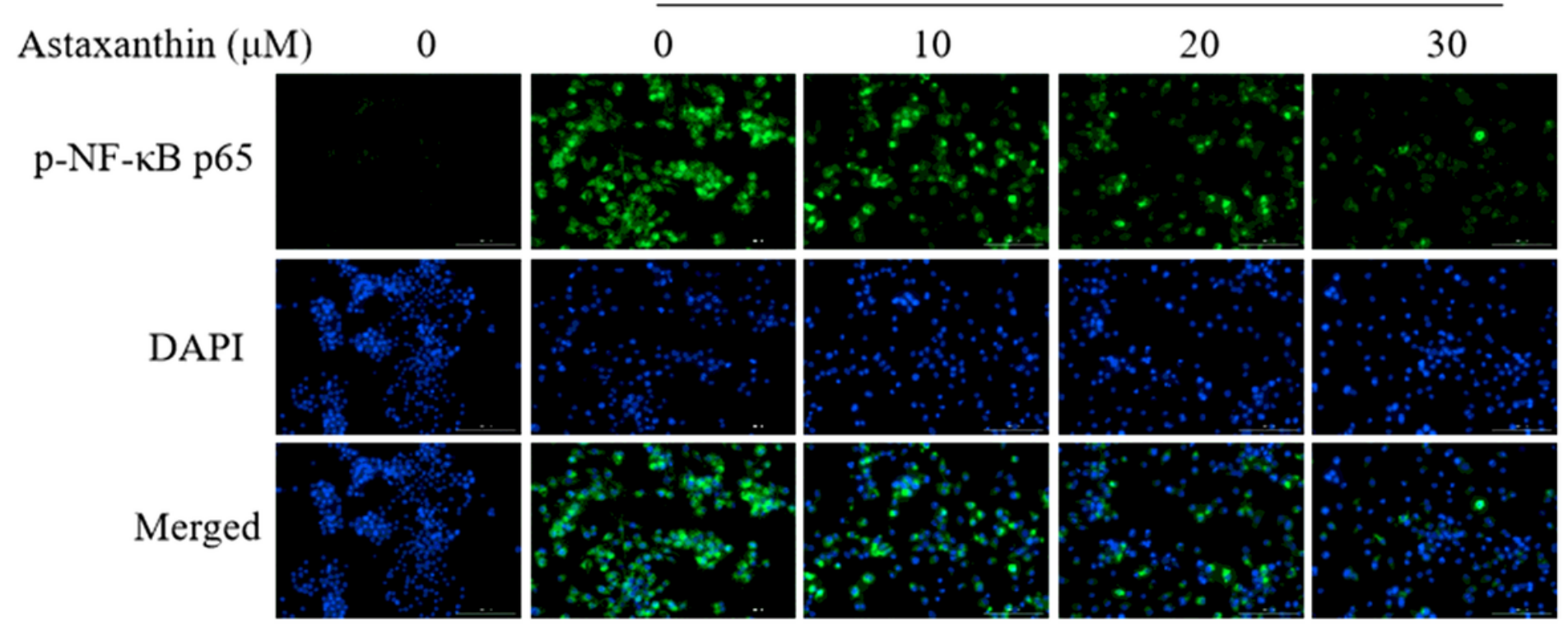

B

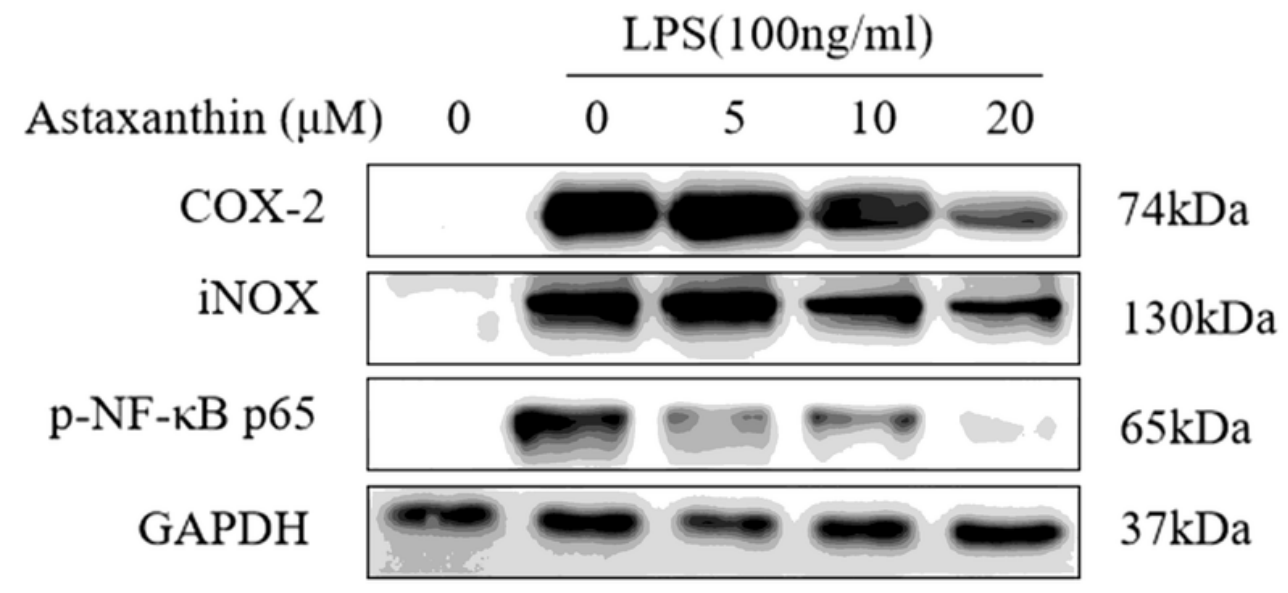

Figure 4

(A) RAW264.7 cells were pretreated with the indicated concentrations of astaxanthin for $1 \mathrm{~h}$ before being stimulated with LPS for another $24 \mathrm{~h}$. COX-2 and iNOS were determined by immunofluorescence staining. DAPI-stained nuclei are indicated by blue fluorescence. COX-2 and iNOS were indicated by green fluorescence. Scale bar $=100 \mu \mathrm{m}$. (B) Cells were pretreated with the indicated concentrations of astaxanthin for $1 \mathrm{~h}$ before being stimulated with LPS $(100 \mathrm{ng} / \mathrm{mL})$ for another $24 \mathrm{~h}$. The expression levels of COX-2 and iNOS were determined by immunoblotting. ${ }^{*} \mathrm{P}<0.05$, ${ }^{\star *} \mathrm{P}<0.01$ compared with the LPS group. 
A

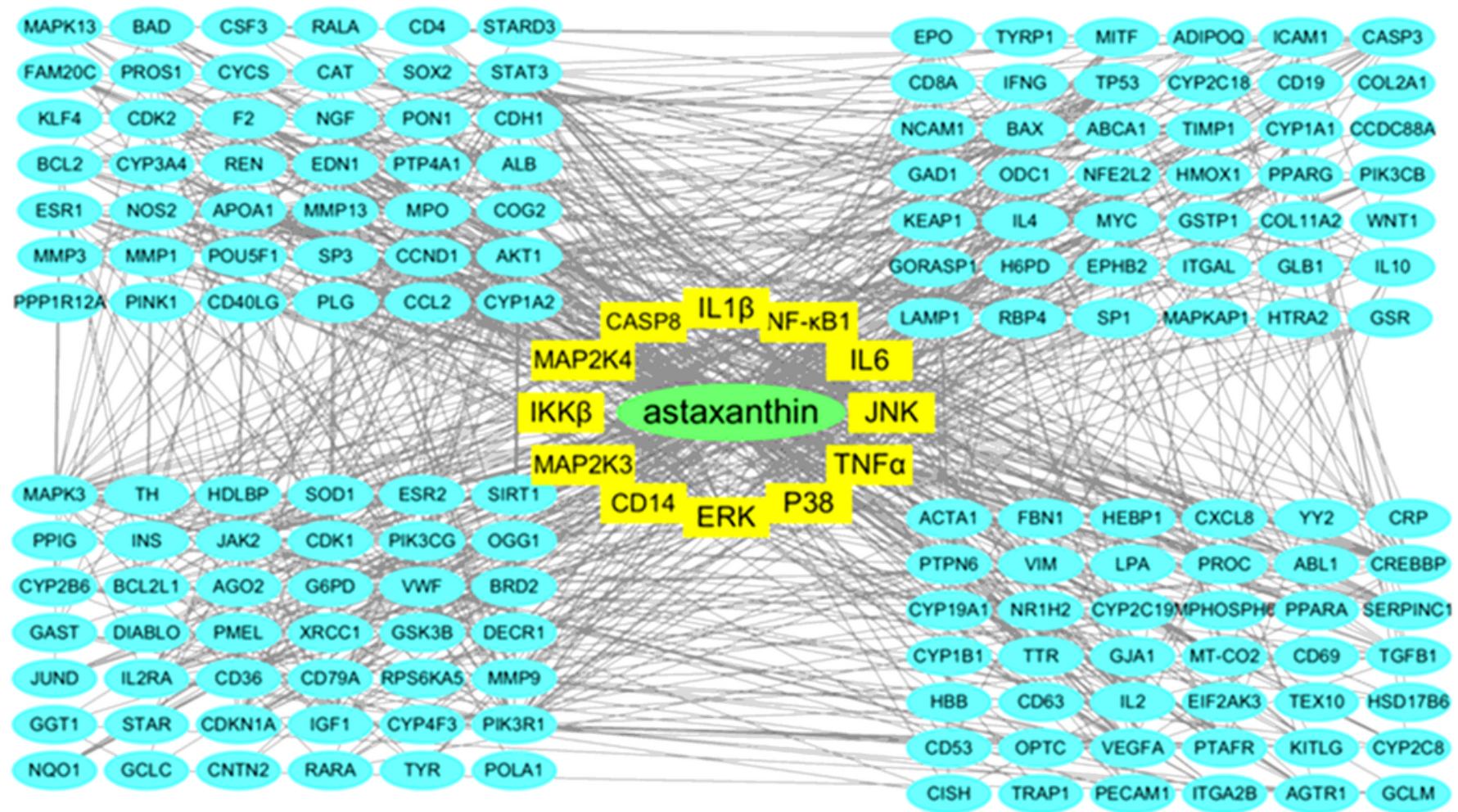

B

\section{LPS(100ng/ml)}

\section{$\operatorname{Astaxanthin}(\mu \mathrm{M})$ \\ $\begin{array}{lllll}0 & 0 & 10 & 20 & 30\end{array}$}

TLR4

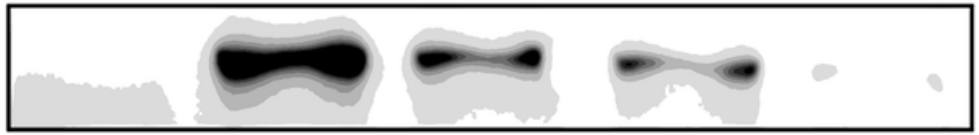

MYD88

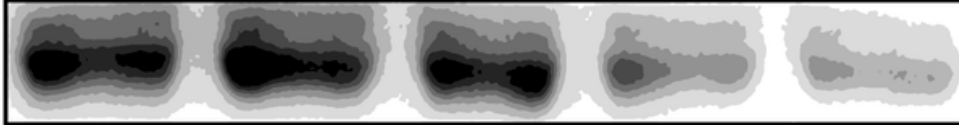

GAPDH

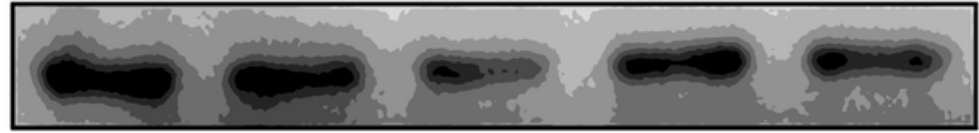

\section{Figure 5}

Astaxanthin inhibits TLR4/MyD88 signaling pathway. (A) Network visualization analysis of TLR4/MyD88 signaling pathways for anti-inflammatory effects of astaxanthin. (B) RAW264.7 Cells were pretreated with the indicated concentrations of astaxanthin for $1 \mathrm{~h}$ before being stimulated with LPS $(100 \mathrm{ng} / \mathrm{mL})$ for another $24 \mathrm{~h}$. The expression levels of TLR4 and MYD88 were determined by immunoblotting. GAPDH was used as a loading control. 
A

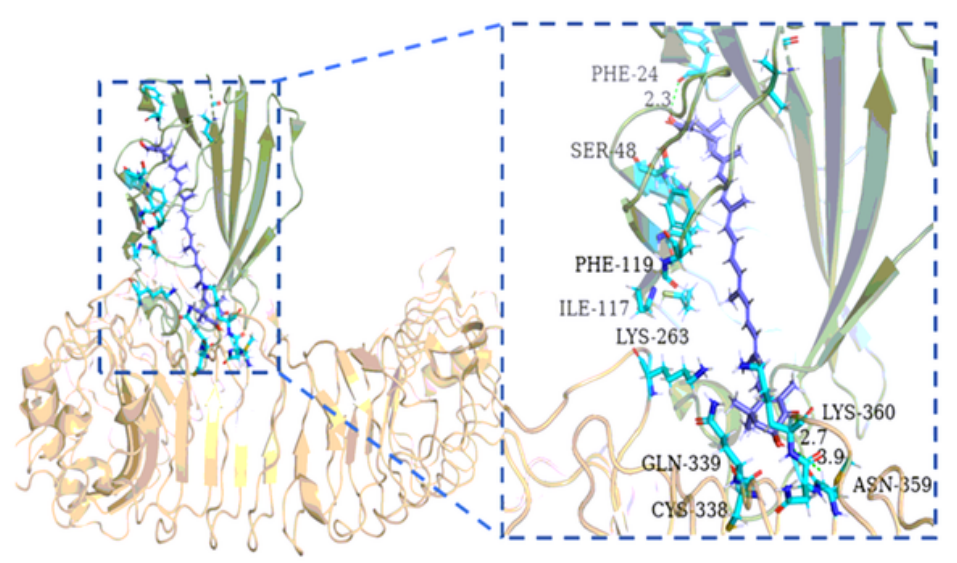

$\mathrm{B}$

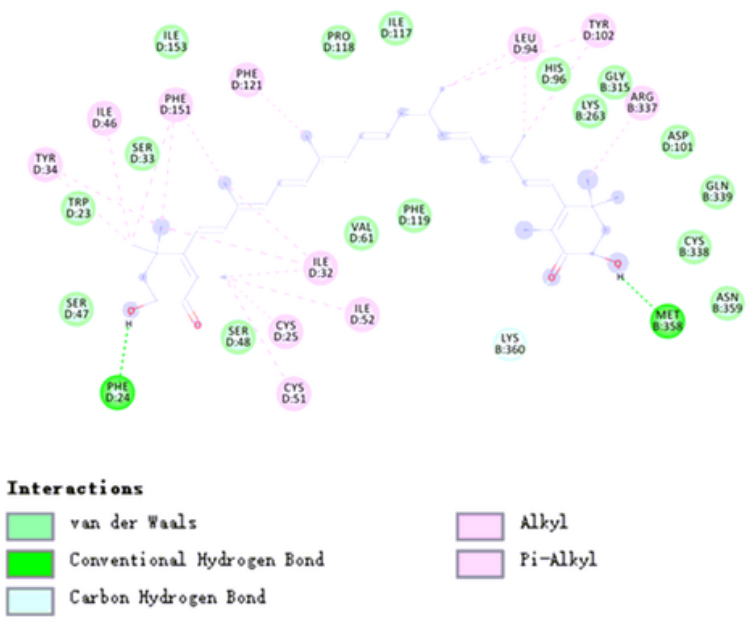

\section{Figure 6}

Computational docking studies of astaxanthin to the TLR4/MD-2 complex. (A) Schematic representations 3D of the binding interactions between the mouse TLR4/MD-2 complex active site and astaxanthin. MD-2 is shown in light green ribbon and TLR4 in light orange ribbon. Close-up view of the predicted interaction between astaxanthin and the MD-2-binding site of the TLR4-MD-2 complex. (B) 2D representation of molecular interactions between the TLR4/MD-2 complex and astaxanthin. 
A

\section{Vehicle}

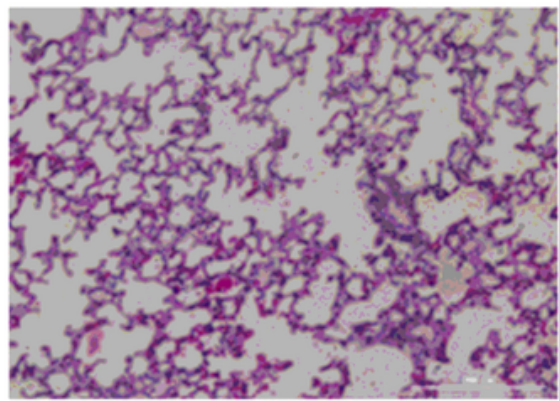

LPS

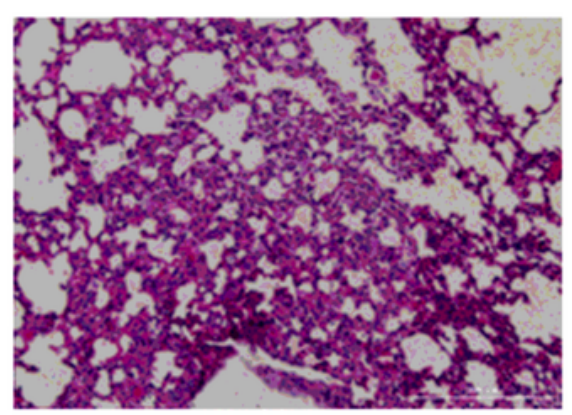

$\mathrm{C}$

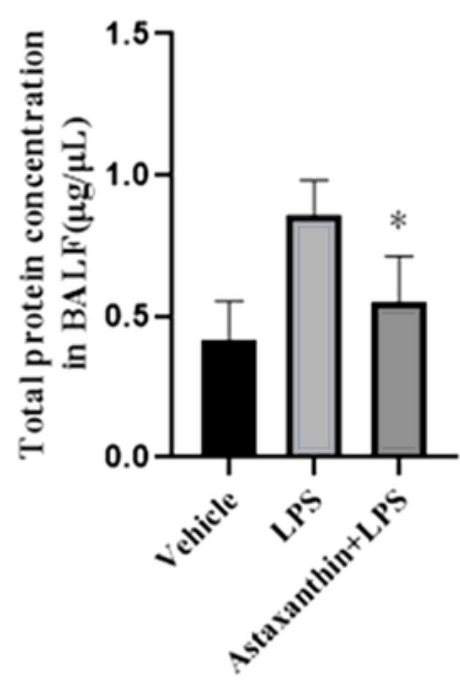

\section{LPS+Astaxanthin}
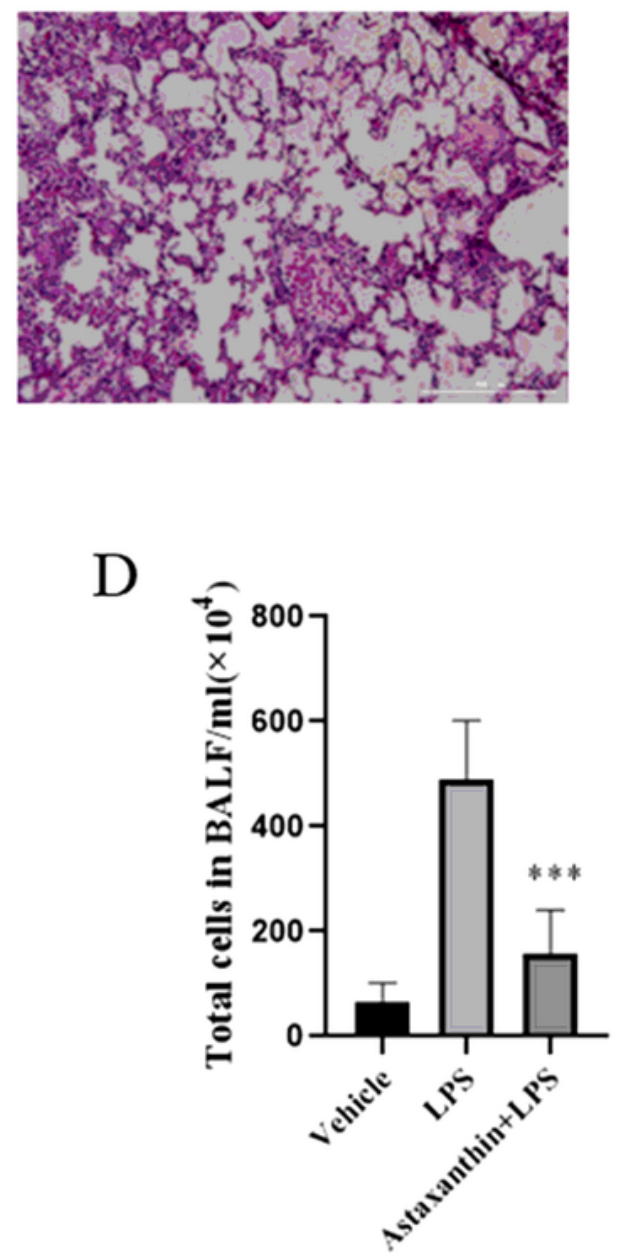

$\mathrm{E}$

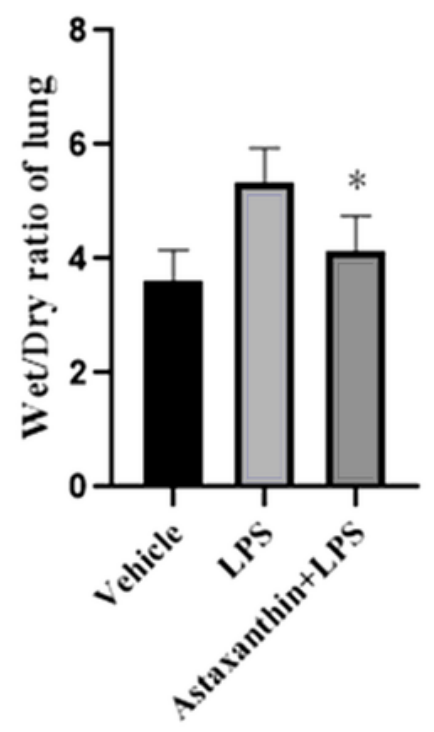

LPS

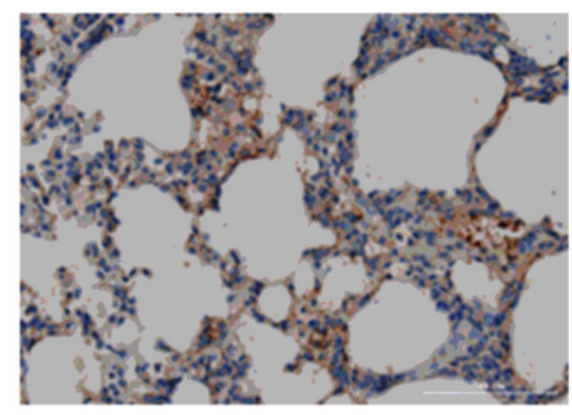

\section{LPS+Astaxanthin}

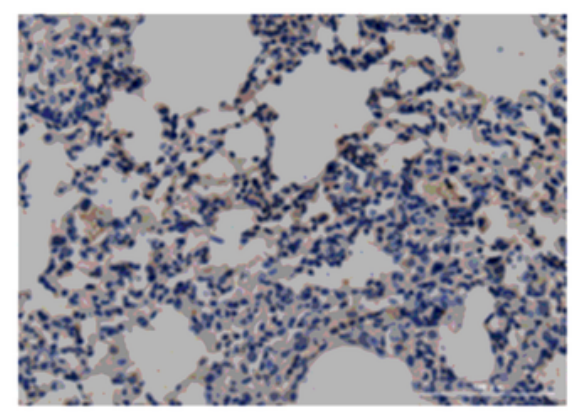

Figure 7

Astaxanthin attenuated the LPS-induced ALI in mice. (A) Representative H\&E staining of lung sections under $200 \times$ magnifications. (B) Wet/dry ratio in the lung tissues. (C) The total protein concentration in BALF. (D) The numbers of total cells in BALF. (E) Representative images of CD68 immunohistochemical staining in the lung sections under $200 \times$ magnifications. Data were presented as mean $\pm S D$. ${ }^{*} P<0.05$, $\star \star \star P<0.001$ vs. LPS group. 


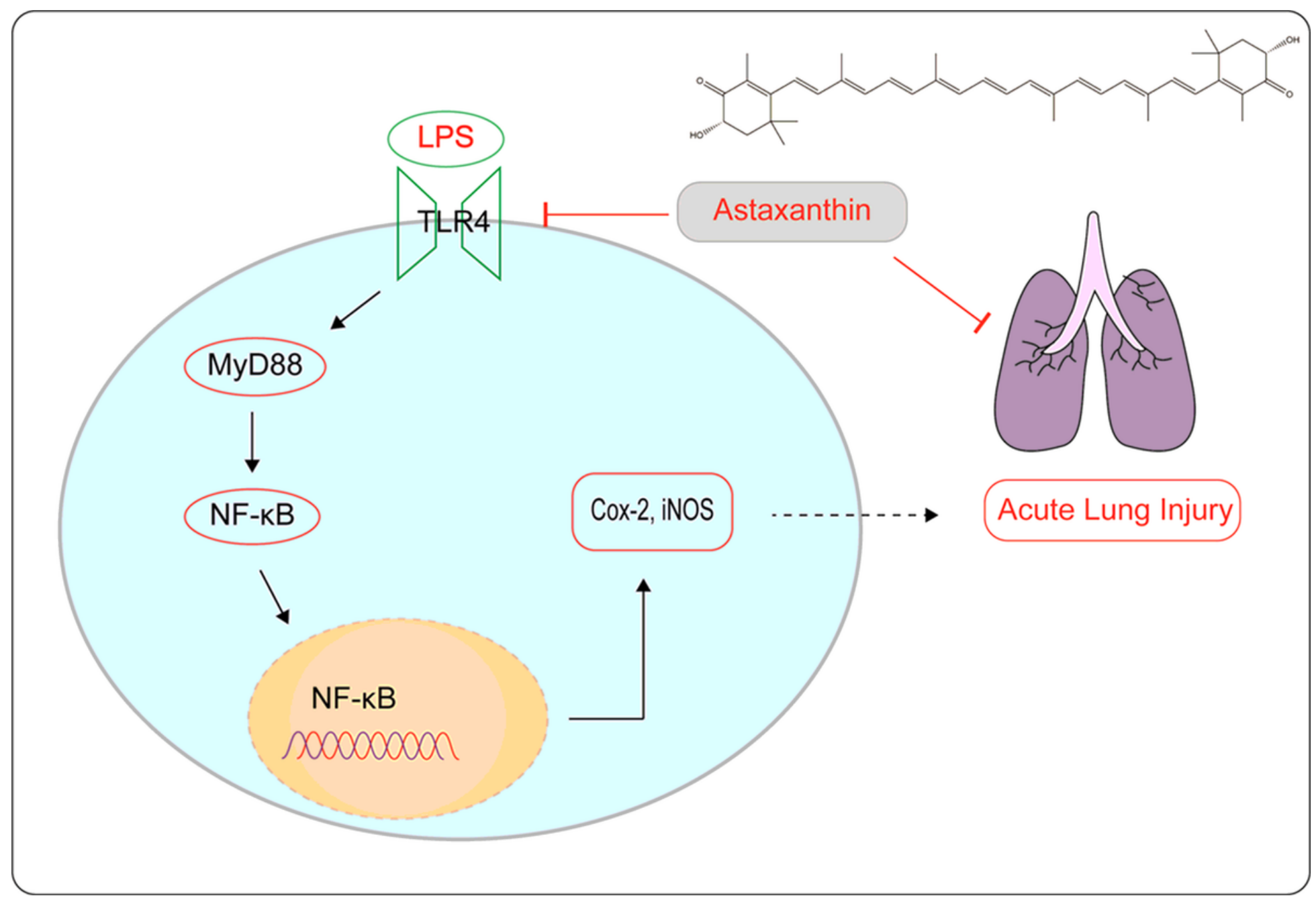

Figure 8

Schematic diagram of signaling pathways related to anti-inflammatory effects of astaxanthin on LPSinduced ALI. 